mid-September 2015, 12 Syrian TB patients were reported to the Netherlands Tuberculosis Register (up until June 30, 2016). Two patients were included in the previous analysis since they had an abnormal chest radiograph at entry screening (one arrived in August 2015 and one in September 2015) and were confirmed by a positive $M$. tuberculosis culture in the fourth quarter of 2015. Six patients arrived in the Netherlands before discontinuation of screening and were diagnosed between 6-14 months after arrival. Four patients arrived in the Netherlands after discontinuation of screening: three of them were diagnosed with pulmonary TB 0-5 months after arrival, and one with pulmonary TB more than 6 months after arrival. If we assume that the three pulmonary TB patients identified after less than 6 months in the Netherlands potentially could have been identified by radiographic screening this would have resulted in a TB prevalence of 18.4 per 100 000 screened persons (95\% CI 4.8-50.0), which is similar to the TB prevalence in our study.

In conclusion, we would like to state that our analysis of entry screening was robust and that close monitoring of the TB situation among Syrian asylum seekers after discontinuation of screening also indicates that their risk for TB is relatively low. The Belgian and Dutch studies on screening (Syrian) asylum seekers show the importance of collecting migrant screening data in a more uniform and systematic way, and reporting on countries' TB screening data and experiences, to better understand screening effectiveness and differences in results between countries. In the recently awarded project, E-Detect TB, by the European Commission several European Union countries are collaborating to pool their screening data together and analyse it [4]. European Union countries not participating in the project will also be invited to join this initiative in a later stage.

$@$ ERSpublications

There is need to collect, analyse and compare TB screening data from migrants in EU countries more systematically http://ow.ly/x2eQ303k2CK

Gerard de Vries ${ }^{1,2}$, Job van Rest ${ }^{1}$, Wieneke Meijer ${ }^{3}$, Henrieke Schimmel ${ }^{2}$, Bert Wolters ${ }^{4}$, Rob van Hest ${ }^{4}$ and Michael Kimerling ${ }^{1}$

${ }^{1}$ KNCV Tuberculosis Foundation, Den Haag, The Netherlands. ${ }^{2}$ Centre for Infectious Diseases Control, National Institute for Public Health and the Environment, Bilthoven, The Netherlands. ${ }^{3}$ Dept of TB Control, Municipal Public Health Services Amsterdam, Amsterdam, The Netherlands. ${ }^{4}$ Dept of TB Control, Regional Public Health Services Groningen, Groningen, The Netherlands.

Correspondence: Gerard de Vries, KNCV Tuberculosis Foundation, P.O. Box 146, 2501 CC Den Haag, The Netherlands. E-mail: gerard.devries@kncvtbc.org

Received: May 032016 | Accepted: June 072016

Conflict of interest: None declared.

\title{
References
}

1 de Vries G, van Rest J, Meijer W, et al. Low yield of screening asylum seekers from countries with a tuberculosis incidence of <50 per 100000 population. Eur Respir J 2016; 47: 1870-1872.

2 Akkerman OW, de Lange WCM, Schölvinck EH, et al. Implementing tuberculosis entry screening for asylum seekers: the Groningen experience. Eur Respir J 2016; 48: 261-264.

3 Ministry of Security and Justice. Immigration and Naturalisation Service (IND). Asylum Trends: Monthly Report on Asylum Applications in The Netherlands and Europe. June 2016. Rijswijk, Ministry of Security and Justice, Immigration and Naturalisation Service (IND), 2016. https://ind.nl/Documents/Asylum\%20Trends\%20June\%202016.pdf

4 E-DETECT TB. https:/e-detecttb.eu/ Date last accessed: August 21, 2016. Date last updated: August 21, 2016.

\section{Comprehensive cost of treating one patient with MDR/pre-XDR-TB in Wellington, New Zealand}

To the Editor:

Diel and co-workers [1, 2] have reported the estimated cost of treating tuberculosis (TB) and multidrug-resistant (MDR)-TB in Germany and the European Union, which, along with estimated and modelling cost data from the USA, Asia and South Africa [3, 4], confirm the huge economic burden to 
range between 40 and 200 times higher than treating fully sensitive TB cases. We describe the full costs of treatment of a person who was treated in 2014-2015 in Wellington, New Zealand. He had MDR-TB plus ofloxacin resistance but marginal moxifloxacin susceptibility (i.e. MDR/pre-extensively drug-resistant (XDR)-TB). This case highlights the key drivers of cost in a high-income country.

In 2013, 276 cases of TB were notified in New Zealand (6.6 per 100000 population); three (1.4\%) cases were MDR-TB [5]. All treatment including hospital admission, investigations and medication for $\mathrm{TB}$ is free to patients in New Zealand through a publicly funded health system.

A 33-year-old Indian male presented with dry cough, malaise and night sweats. Born in India, he immigrated to New Zealand 6 years earlier. Unemployed, with no prior history of TB, he presented with a body mass of $56 \mathrm{~kg}$, fever, sinus tachycardia, cervical lymphadenopathy, a small pleural effusion and delusional ideation. Radiology confirmed pulmonary, pleural and mediastinal lymph node changes consistent with TB. An HIV test was negative. Sputum, induced sputum, pleural fluid aspirate, lymph node aspirate, cerebrospinal fluid and urine were direct smear negative for acid-fast bacilli (AFB), as were subsequent bronchoscopy washings, but a post-bronchoscopy sputum sample showed two or more AFB. Daily antituberculous therapy (ATT) was started with isoniazid, rifampicin, pyrazinamide and ethambutol, but there was persistence of systemic symptoms after 3 weeks' treatment. Genotypic resistance testing (GeneXpert (Cepheid, Banksmeadow, Australia) and HAIN (Hain Lifescience GmbH, Nehren, Germany)) and susceptibility testing (BACTEC MGIT 960 SIRE (BD, Franklin Lakes, NJ, USA)) was performed in local and national reference laboratories. Resistance was demonstrated to isoniazid (high level), rifampicin, para-aminosalicylic acid (PAS) and ethionamide in both laboratories. Fluoroquinolone susceptibility testing provided discordant results for ofloxacin (resistant) and moxifloxacin (marginal susceptibility, MIC90 $2 \mathrm{mg} \cdot \mathrm{L}^{-1}$ ). Susceptibility to pyrazinamide, ethambutol, amikacin and linezolid was demonstrated. Genotypic resistance mutations included isoniazid (katG), rifampicin (rpoB S531 L), ethambutol (M3601) and moxifloxacin/ofloxacin (gyrA D94G).

\begin{tabular}{|c|c|c|c|}
\hline Item & Unit cost NZD & Units & Total NZD \\
\hline \multicolumn{4}{|l|}{ Inpatient costs } \\
\hline Bed day cost ${ }^{\#}$ & 1653 per day & 99 days ${ }^{\Uparrow}$ & 163647 \\
\hline Radiology and laboratory ${ }^{+}$ & & & 11367 \\
\hline Total inpatient costs & & & 175014 \\
\hline \multicolumn{4}{|l|}{ Outpatient costs ${ }^{\S}$} \\
\hline Outpatient medical including all tests ${ }^{f}$ & & 15 visits & 6844 \\
\hline Public health nurses $\# \#$ & 75.80 per visit & 541 visits & 41008 \\
\hline District nurses & 121.68 per visit & 287 visits & 34922 \\
\hline Total outpatient costs & & & 82774 \\
\hline Interdisciplinary individual case management meetings ${ }^{++}$ & 300 & 12 meetings & 3600 \\
\hline \multicolumn{4}{|l|}{ Medications ${ }^{\S \S}$} \\
\hline \multicolumn{4}{|l|}{ First line } \\
\hline Isoniazid/rifampicin ${ }^{f f}$ & & & 121 \\
\hline Pyrazinamide & & & 1132 \\
\hline Ethambutol & & & 1401 \\
\hline Total first-line medication costs & & & 2654 \\
\hline \multicolumn{4}{|l|}{ Second line } \\
\hline Amikacin & & & 27942 \\
\hline PAS & & & 12043 \\
\hline Moxifloxacin & & & 6289 \\
\hline Clofazamine & & & 4500 \\
\hline Linezolid & & & 11941 \\
\hline Total second-line medication costs & & & 62715 \\
\hline Total medication costs & & & 65369 \\
\hline Total cost of treatment & & & 326757 \\
\hline
\end{tabular}

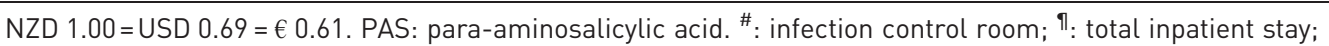
${ }^{+}$: biochemistry, haematology, viral panel, amikacin serum levels, computed tomography (CT) of the chest, CT head, bronchoscopy and tuberculosis (TB) testing, audiometry, and vision tests; ${ }^{\S}$ : excluding medication: $f_{\text {: }}$ biochemistry, haematology, amikacin serum levels, follow-up sputum TB testing, audiometry,

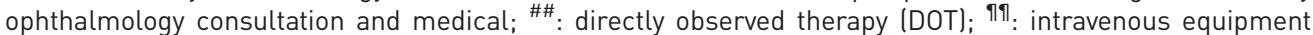
and DOT at weekends and on public holidays; ${ }^{++}$: prior to and after discharge, ensuring successful transition to the community; ${ }^{\S \S}$ : inpatient and outpatient; ${ }^{f f}$ : first 4 weeks only. 
In accordance with the World Health Organization (WHO) consensus approach to regimen make-up [6], second-line ATT comprised daily intravenous amikacin, oral moxifloxacin, PAS, linezolid and clofazimine in addition to pyrazinamide and ethambutol. PAS was included on expert recommendation despite the sensitivity result. Cycloserine was avoided because of the psychiatric comorbidity. Bedaquiline was available on compassionate grounds but not prescribed given the increased risk of QTc prolongation when co-administered with moxifloxacin, clofazimine and antipsychotic medication [7].

Induced sputum and pleural aspirate samples taken at 6 weeks after starting second-line agents were TB culture negative, and he was discharged from hospital after 99 days. The hospital stay was complicated by the new diagnosis of paranoid schizophrenia with depression requiring treatment with olanzapine, aripiprazole and sertraline.

The patient developed hearing impairment, presumed due to amikacin, necessitating a shortened intravenous course (5.75 months). Treatment was stopped at 17 months when he developed severe bilateral visual impairment. Follow-up, 6 months after treatment cessation, confirmed he was well, with a body mass of $68 \mathrm{~kg}$, and his (induced)sputum culture remained negative. Visual acuity had recovered to 6/9 bilaterally, hearing was sufficient for ordinary conversation without augmentation and mental health problems were stable on treatment.

Treatment costs were retrospectively obtained from the District Health Board and counted in New Zealand dollars $($ NZD $1.00=$ USD $0.69=€ 0.61)$, detailed in table 1 .

Reported estimated and modelled costs for treating MDR-TB range from $€ 1097$ to $€ 75066$ [1-4], correlating with country's wealth, and influenced by variability of local public health policies, pharmacological prices, and patient comorbidities and drug side-effects.

The cost of treating this patient in New Zealand was almost NZD 327000 (approximately USD 225 000/ $€ 200000)$. The cost for treating fully susceptible TB in New Zealand was previously estimated at NZD 17000 per patient [8]. Most of the cost in this case was from an extended inpatient stay (54\% of total, 99 days), the large number of domiciliary visits for directly observed therapy, and intravenous therapy $(23 \%$ of total). Medication costs were significant, particularly for second-line agents (19\% of total patient cost).

The prolonged inpatient stay reflected concerns about fluoroquinolone susceptibility, concordance with treatment given the acute psychiatric illness and regional public health policy. Inpatient days could have been reduced by up to 3 weeks (saving up to NZD 34700) if genotypic resistance testing had been performed on the initial samples. We estimate that his psychiatric comorbidity inflated costs by a maximum of NZD 16530 (up to 10 extra inpatient days), together reducing the total to NZD 275527 (€ 168000).

The extreme, but affordable, cost of this single case highlights the global challenge of the WHO End TB plan [9] to mitigate the financial consequences of the treatment of MDR-, pre-XDR- and XDR-TB on the health systems of low-resource countries as well as for the patients themselves [10].

0 @ERSpublications $€ 200000$ : the full cost of treating a single MDR/pre-XDR-TB patient in New Zealand http://ow.ly/NkGT301B5KN

Amanda McNaughton ${ }^{1,2}$, Timothy Blackmore ${ }^{3}$ and Harry McNaughton ${ }^{2}$

${ }^{1}$ Dept of Respiratory Medicine, Capital and Coast District Health Board, Wellington, New Zealand. ${ }^{2}$ Dept of Infectious Diseases, Capital and Coast District Health Board, Wellington, New Zealand. ${ }^{3}$ Medical Research Institute of New Zealand, Wellington, New Zealand.

Correspondence: Amanda McNaughton, Dept of Respiratory Medicine, Capital and Coast DHB, Private Bag 7902, Wellington South 6021, New Zealand. E-mail: amanda.mcnaughton@ccdhb.org.nz

Received: May 032016 | Accepted: June 072016

Conflict of interest: None declared.

\section{References}

1 Diel R, Vandeputte J, de Vries G, et al. Costs of tuberculosis disease in the European Union: a systematic analysis and cost calculation. Eur Respir J 2014; 43: 554-565.

2 Diel R, Rutz S, Castell S, et al. Tuberculosis: cost of illness in Germany. Eur Respir J 2012; 40: 143-151.

3 Laurence YV, Griffiths UK, Vassall A. Costs to health services and the patient of treating tuberculosis: a systematic literature review. Pharmacoeconomics 2015; 33: 939-955.

4 Pooran A, Pieterson E, Davids $\mathrm{M}$, et al. What is the cost of diagnosis and management of drug resistant tuberculosis in South Africa? PLoS One 2013; 8: e54587.

5 Institute of Environmental Science and Research Ltd. Tuberculosis in New Zealand: Annual Report 2013. Wellington, ESR, 2015. 
6 Falzon D, Jaramillo E, Schünemann HJ, et al. WHO guidelines for the programmatic management of drug-resistant tuberculosis: 2011 update. Eur Respir J 2011; 38: 516-528.

7 Pontali E, Sotgiu G, D’Ambrosio L, et al. Bedaquiline and multidrug-resistant tuberculosis: a systematic and critical analysis of the evidence. Eur Respir J 2016; 47: 394-402.

8 Burns AD, Harrison AC. Costs of investigating and managing non-residents with possible tuberculosis: New Zealand experience of an international problem. Respirology 2007; 12: 262-266.

9 World Health Organization. WHO End TB Strategy Global Strategy and Targets for Tuberculosis Prevention, Care and Control after 2015. Geneva, WHO, 2015.

10 Stout JE. The cost of "free" tuberculosis care. Int J Tuberc Lung Dis 2015; 19: 1413.

Eur Respir J 2016; 48: 1256-1259 | DOI: 10.1183/13993003.00876-2016 | Copyright (C)ERS 2016 